\title{
Phase Modulation Characteristics of Spatial Light Modulator and the System for Its Calibration
}

\author{
Zhang Gongjian ${ }^{1}$, Zhang Man ${ }^{1}$ and Zhao Yang ${ }^{2}$ \\ 1. Department of Opto-Electronic Engineering, Chitose Institute of Science and Technology, Chitose 066-8655, Japan \\ 2. Xi'An Jiaotong University City College, No. 8715 Shangji Road, Xi'an 710018, Shaanxi Province, China
}

\begin{abstract}
The phase modulation characteristics of liquid crystal SLM (spatial light modulator) and the system for calibrating are proposed. Michelson interferometer is employed for measuring the modulation properties of device. A system interface for operating SLM is designed with $\mathrm{VC}++$ compiler. The initial distortion phase is determined by measuring the reflective interference, and modulation of device is compensated by using our system. By using the LUT (lookup table) data provided by manufacture the wavelength disperse calibration is also achieved successfully.
\end{abstract}

Key words: liquid crystal SLM, phase modulation, wavefront correction, adaptive optics.

\section{Introduction}

The advent of SLMs (spatial light modulators) has contributed enormously to various applications in photonics during the last years. The device is regarded as a perfect wave-front controller because of its advantages such as low-power consumption, high-resolution, non-mechanical and programming control. In general, SLM is applied to modulate amplitude, phase or polarization of a light wave. However, reliable application of SLMs as programmable diffractive optical elements requires a thorough calibration, because the individual SLM from manufacture has its inherent modulation property. Therefore, there are many problems encountered in practical applications, such as the need for an easy-to-use interface, an inexpensive and precise calibration and measurement system, and a series of reliable and stable algorithms for phase extraction. All of these are problems to be solved. In view of these problems, this study attempts to explore and propose a complete set of solutions to many problems encountered by SLM in practical application from

Corresponding author: Gongjian Zhang, associate professor, research fields: information photonics, electrical and optical material science. theoretical algorithm to experimental method. At first, the phase modulation characteristics of liquid crystal SLM and the system for calibrating are proposed. Michelson interferometer is employed for measuring the modulation properties of device. A system interface for operating SLM is designed with $\mathrm{VC}++$ compiler. Then, the initial distortion phase is determined by measuring the reflective interference and modulation of device is compensated by using our system. At the same time, by using the LUT data provided by manufacture the wavelength disperse calibration is also achieved successfully.

\section{Mathematical Analysis and Modulation Manner}

The experimental arrangement is shown in Fig. 1. It is based on a Michelson interferometer adapted for polarization phase shifting. With a He-Ne laser $(\lambda=$ $632.8 \mathrm{~nm})$ as the source, a spatially filtered and expanded collimated beam is obtained in the usual manner as shown in Fig. 1.

The polarizers $\mathrm{P} 1, \mathrm{P} 2$ and quarter wave plate are combined, so that the reference beam (reflected from the mirror) and the sample beam (reflected from the SLM) maintain their appropriate polarization states on 


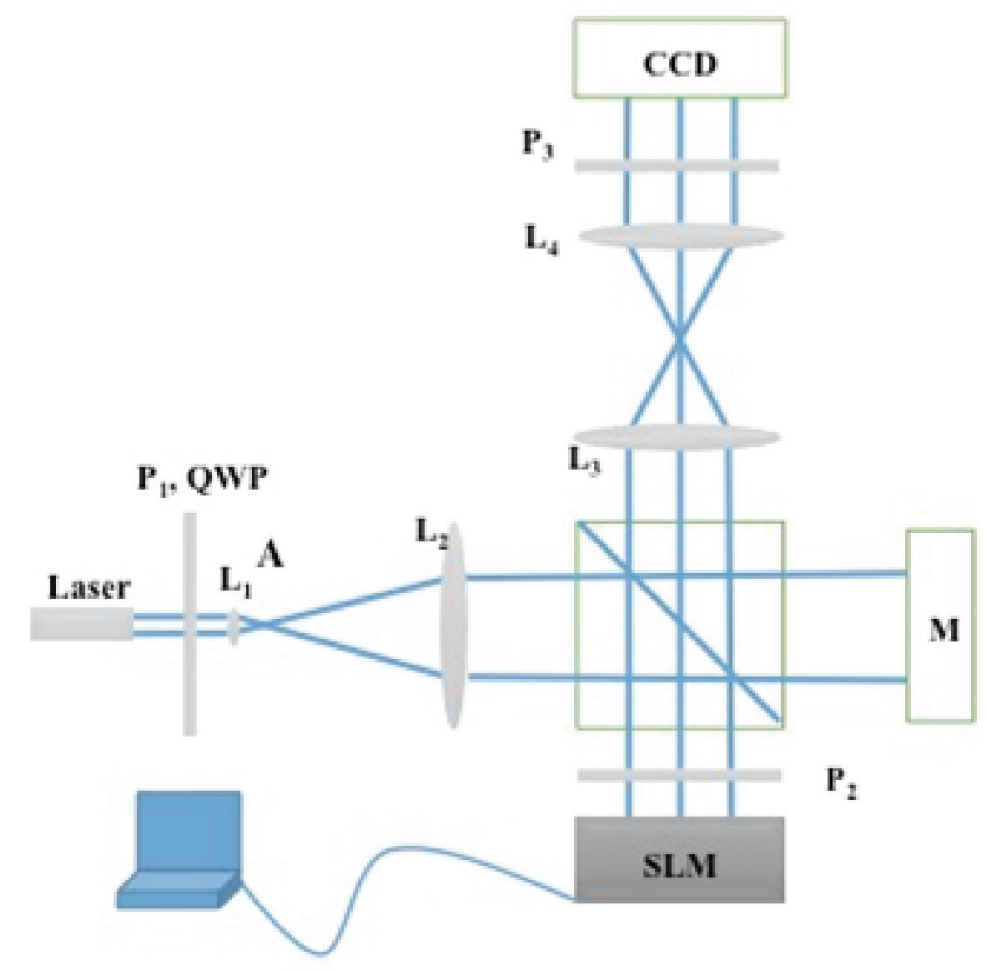

Fig. 1 Optical test setup for analyzing SLM modulation properties.

emerging from the cubic beam splitter. A polarizer P3 is introduced to make the two beams interfere. CCD (Charge-coupled device) is located in the back focal plane of L4. With this arrangement the interferogram was recorded.

The test system is a reflective SLM that can be equivalently unfolded in the form of a cascade of two identical transmissive cells with mirror-symmetric structures. Using the convenient Jones matrix formulation, the amplitude of the propagating wave front reflected from the SLM after the final polarizer can be expressed as Ref. [1].

$$
J=\exp (-i 2 \beta)\left[\begin{array}{cc}
(\alpha / \gamma)^{2}+(\beta / \gamma)^{2} \cos (2 \gamma)-i \beta \sin (2 \gamma) / \gamma & i \alpha \beta[1-\cos (2 \gamma)] / \gamma^{2} \\
i \alpha \beta[1-\cos (2 \gamma)] / \gamma^{2} & (\alpha / \gamma)^{2}+(\beta / \gamma)^{2} \cos (2 \gamma)-i \beta \sin (2 \gamma) / \gamma
\end{array}\right]
$$

In this case, the complex amplitude reflectance and phase shift of the entire devices are as followings:

$$
\begin{aligned}
& R=\left\{\left[(\alpha / \gamma)^{2}+\right.\right.\left.\left.(\beta / \gamma)^{2} \cos (2 \gamma)\right] \cos \left(\psi_{1}-\psi_{2}\right)\right\}^{2} \\
&+\left\{\frac{\alpha \beta}{\gamma^{2}}[1-\cos (2 \gamma)] \sin \left(\psi_{1}+\psi_{2}\right)-\frac{\beta}{\gamma} \sin (2 \gamma) \cos \left(\psi_{1}-\psi_{2}\right)\right\}^{2} \\
& \delta=2 \beta-\tan ^{-1}\left\{\frac{\frac{\alpha \beta}{\gamma^{2}}[1-\cos (2 \gamma)] \sin \left(\psi_{1}+\psi_{2}\right)-\frac{\beta}{\gamma} \sin (2 \gamma) \cos \left(\psi_{1}-\psi_{2}\right)}{\left[(\alpha / \gamma)^{2}+(\beta / \gamma)^{2} \cos (2 \gamma)\right] \cos \left(\psi_{1}-\psi_{2}\right)}\right\}^{2}
\end{aligned}
$$


In general, the device is in both amplitude and phase modulated of coupling manner. The phase only or most amplitude modulation can be achieved by selecting an appropriate configuration. For example, if $\psi_{1}=\psi_{2}=0$, smaller $\beta$ value range can be an amplitude modulation, and larger $\beta$ value will be in phase modulation.

In LCOS SLM, there are two modulation modes: amplitude and phase modulation. In general, these two modes are in a coupled state. However, if appropriate parameter conditions are selected, a simple modulation mode with only amplitude modulation or phase modulation can be obtained. Using Eqs. (2) and (3), as a function of parameter $\beta$, we can obtain the transmittance $T$ and the phase modulation quantity $\delta$. In Fig. 2, we show the dependence relation between the transmittance $\mathrm{T}$ and the phase modulation measure $\delta$. $\delta$ is relative to the beta parameter (the polarization angle of incident light and transmitted light is 0 ). It is important to note that the phase in Eq. (3) is represented by the inverse trigonometric function, so the obtained phase is often the wrapped phase. In order to obtain the real phase, the wrapped phase should be unwrapped. As shown in Fig. 2, in the case of smaller values of the beta (in the figure, between 0 and square root three $\mathrm{PI} / 4$ ), the phase change range is very small, the transmission rate is basically changed linearly with the beta, and so the amplitude modulation can be obtained in this range. On the other hand, in the case of large beta (in the diagram, greater than the square root of $3 \mathrm{PI} / 4$ ), the change of the transmittance is small, and the phase changes basically just follow the applied voltage, so the modulation of this case will be the phase modulation. If the parameters are set properly, the pure phase modulation or pure amplitude modulation can be realized.

\section{Experimental Principle}

In this section, the algorithms for phase measuring, phase extracting, and phase unwrapping will be summarized. All of these functions will be integrated into this SLM operating application.

\subsection{Phase Recovery from Interferogram}

(1) PSI (phase-shifting interferometry) method

PSI method has drawn much attention in many areas of applications because of its high precision in optical measurements. This method utilizes the temporal domain to collect a series of interferograms by

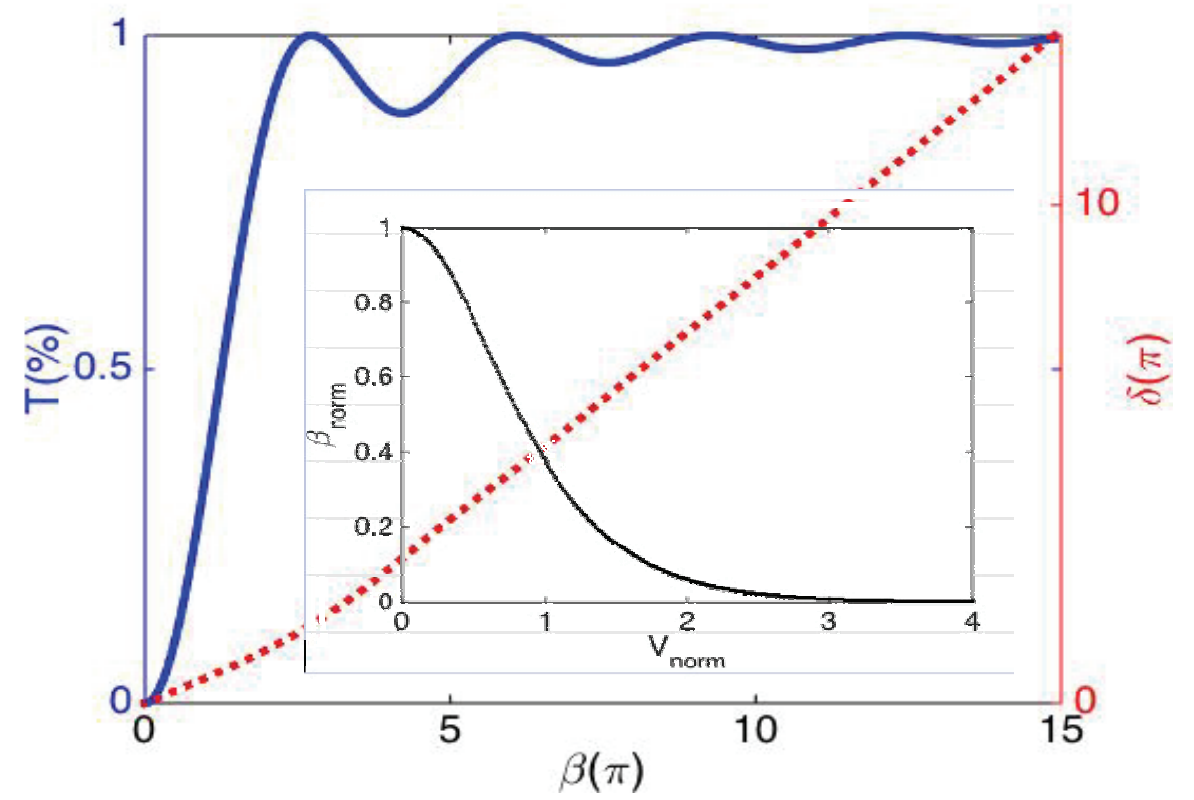

Fig. 2 The relationship between phase modulation $\delta$, transmission $T$, and parameter $\beta$. 
adjusting the phase of the reference beam. To create multiple interferograms, a method is needed to introduce the required phase shift in the reference beam. The most commonly used method is a movable mirror driven by a PZT (piezoelectric transducer) to induce a path-length change in the reference beam. A similar effect is achieved by translating a prism orthogonal to the beam. Other methods which have also been used to introduce a phase shift include translating diffraction grating perpendicularly to the light beam and rotating polarization phase retarders. In all these phase-shifting methods, the accuracy and stability of the motion of the phase-shifter is critical to the accuracy of optical measurements. Thus the accuracy and stability of the reference phase-shifter poses one of the most serious limitations for precision phase measurement in PSI systems.

(2) The Fourier transform method

The optical flatness of reflecting devices, such as mirrors and LCOS-SLMs surface, can be easily measured by interferometer. In optical measurement of the interferometer, the interferogram fringe patterns are of the form $\mathrm{I}=\mathrm{a}(\mathrm{x}, \mathrm{y})+\mathrm{b}(\mathrm{x}, \mathrm{y}) \cos \left(\varphi_{\mathrm{r}}+\varphi_{0}\right)$, here, $\varphi_{\mathrm{r}}$ and $\varphi_{0}$ express the reference and initial phase distributions respectively.

In order to get the quantitative evaluation of co-sinusoidal fringe patterns obtained from an optical interferometer, the FFT transformation method as the signal processing technique is employed commonly.

Its advantage is that just single frame of interferogram is needed for extracting the phase information.

In this case, usually a carrier was introduced into a normal interference fringe pattern. It is similar to the carrier modulation in the communication technology, here the examples as usual are Moiré and Fringe Projection Techniques.

The interference patterns were then simulated from these modeled phase values according to following expressions:

$$
\begin{array}{r}
\mathrm{I}(\mathrm{x}, \mathrm{y})=\mathrm{a}(x, y)+b(x, y) \cos \left[\varnothing(x, y)+2 \pi f_{0} x\right]= \\
a+c e^{i 2 \pi f_{0} x}+c * e^{-i 2 \pi f_{0} x}
\end{array}
$$

where $\mathrm{I}(\mathrm{x}, \mathrm{y})$ is the intensity distribution in the detection plane $(\mathrm{x}, \mathrm{y}), \mathrm{a}(\mathrm{x}, \mathrm{y})$ denotes the mean intensity of the interference field, $b(x, y)$ characterizes the modulation of the detected interference signal, $f=$ (fx, fy) is the spatial carrier frequency, $\mathrm{N}_{\mathrm{m}}$ and $\mathrm{N}_{\mathrm{a}}$ characterize multiplicative and additive noise in the interference pattern. When the carrier frequency is large enough, the interference fringes with opened loop are obtained. We can use the Fourier transform to demodulate the interferogram and extract the phase information.

Here, $c=(1 / 2) b(x, y) e^{i \varphi(x, y)}$, the symbol “*” means complex conjugate. According to the basic properties of the linear and frequency shift of the Fourier transform, you can get the spectrum as follows,

$\tilde{\mathrm{I}}(\mathrm{u}, \mathrm{v})=\tilde{\mathrm{a}}(u, v)+C\left(u+f_{0}, v\right)+C^{*}\left(u-f_{0}, v\right)(5)$ where, $\widetilde{\mathrm{a}}(u, v)$ is a narrow peak at the center of the Fourier spectrum, representing the spectrum of the background and the slowly varying amplitude. And $C\left(u+f_{0}, v\right)$ and $C^{*}\left(u-f_{0}, v\right)$ are two spectra of the interferogram, which are complex conjugates of each other, and they are symmetric relative to the origin of frequency.

The phase extraction is to isolate the spectrum $\mathrm{C}$ or spectrum $C^{*}$, and to carry out the inverse Fourier transform, the package phase of the interferogram can be obtained.

In summary, the Fourier transform method is based on the Fourier transform of the distribution of the intensity of the interference field. The two-dimensional Fourier transform of the interference pattern is a Hermitian function and the amplitude spectrum thus looks symmetric with respect to the dc-term. The spectral peak at zero frequencies represents low frequency spectral component that arises from the modulation of the background intensity of the interferogram.

Two symmetric spectral side lobes carry the same 
information about the phase values $\varphi_{\mathrm{r}}$. Using appropriate adaptive band pass filters in the spatial frequency domain we can extract one of side lobes. The wrapped phase values can be then calculated by the inverse Fourier transform of the filtered spectrum. However, there are still many difficult problems to solve.

(i) Because being a wrapped phase, discontinuities of phase values must be correctly unwrapped by appropriate mathematical techniques.

(ii) In order to select appropriate spectrum and eliminate the noise and background of interferogram, the filtering technique is employed. However, the badly chosen filter may cause a coarse disturbance of resulting data.

(iii) For the phase extraction from an interference pattern in which carrier was introduced, it is necessary to eliminate the phase tilt after phase recovery. This is because when the spectrum is selected by filtering technology, even small errors in the spectrum can cause relatively large phase tilt.

(3) RQPT (regularized quadrature and phase tracking) method.

As mentioned above, in the method of carrier interference fringe measurement using FFT analysis, large errors may occur when the fringe frequency is low. If the surface of the test is undulating, it is not even possible to obtain the carrier interference fringes.

In addition, in the fringe analysis method based on FFT, the object of parsing is just the brightness of the interference fringe information, in this way, because the target light phase and phase conjugate and $\mathrm{dc}$ component exist at the same time, so still it cannot get the required phase information directly.

It is necessary to separate the required information with the help of filtering technology to extract the phase. Therefore, this method is not guaranteed to be effective in most cases.

In addition, as the traditional optical interference measuring method of phase shift method, it is changing the measured surface and the relative distance between the interferometer reference surface at the same time, to capture multiple interference image, and to estimate the surface shape from the information. However, it is difficult to guarantee the stability of the instrument and reduce the cost of the measuring instrument.

In this method, since it is necessary to capture a plurality of images, there is a problem that accuracy deteriorates greatly in an environment with disturbance such as vibration.

In view of the above, there is a growing need to measure the phase accuracy from the one-shot interference pattern of the closed fringe that is not restricted by the local frequency of the fringes. In this section we discuss phase recovery from closed fringe interference patterns.

In the past few years, lots of well-known techniques for phase extraction from a single interferogram, which possess closed fringe, have been developed [2-4]. One of the most successful approaches is the RPT (regularized phase tracking) technique, which is robust and powerful enough to demodulate many kinds of fringe pattern, for example, both open and closed noisy fringe patterns. In addition, it does not need further phase unwrapping processing. The typical examples are as RPT, PIRPT, GRPT, RPT method, often fail in the cases of complex interferograms and have relatively low phase reconstruction accuracy. For the PIRPT method, it neglects the fringe background and modulation term. For the method of GRPT/iGRPT, because dependence of scanning path and fringe density were improved, it was found that this method is efficient for demodulating many patterns of fringe. The method in our research is based on the RPT and GRPT/iGRPT. It was improved for unwrapping the estimated phase. We take into account the following 3 factors:

(i) Initial iteration value setting, it can realize eliminating ambiguity of the solution, and obtain a unwrapped phase;

(ii) Phase front approximation using Taylor 
polynomial up to third order differential, it may make the iteration initial value setting more reasonable.

(iii) Optimal cost function consist of the terms of least square optimization, regularization, and quadrature of fringe pattern, making the cost function symmetry to improve the reliability so that almost all fringe patterns including both open and closed fringe pattern, could be demodulated and do not need any further unwrapping processing.

\subsection{Phase Unwrapping}

In our operating system, it is possible to use a measured distortion phase at a fixed wavelength combining with the LUT provided by manufactory for the calibration of the wavelength dispersion. However, the phase value obtained is usually wrapped between $-\pi$ and $\pi$. If one wants to use the LUT coefficient to correct the wavelength disperse, the correct phase value is needed. Next procedure is phase unwrapping processing [5-9]. The relationship between wrapped phase $\psi$ and unwrapped phase $\varphi$ can be expressed as $\psi_{\mathrm{ij}}=\varphi_{\mathrm{ij}}+2 \pi \mathrm{k}$, in which $k$ is integer. The relation can be exchanged as following form.

$$
\varphi_{i+1, j}+\varphi_{i-1, j}+\varphi_{i, j+1}+\varphi_{i, j-1}-4 \varphi_{i, j}=\rho_{i, j}
$$

Here, the $\rho_{i, j}$ expresses the differential of the phase difference at i, j position. Finally, a Poisson's equation derived from this differential scheme can be solved by a method of the fast discrete cosine transform and an unwrapped profile of the phase distribution can be obtained.

When the initial distortion phase of SLM is extracted based on the Fourier spectrum analysis method of interference fringes, the inclined plane is often introduced in the phase surface data. To eliminate the tilt in this phase data, it is necessary to unwrap the wrapped phase data.

In this case, the initiation process requires iteration. However, the noise in the phase data can cause distortion in the iteration calculation. This will greatly affect the unwrapping precision. In this case, FFT or DCT (discrete cosine transform) method has the function of noise filtering. When the iterative calculation is not sufficient, the low-frequency components are very distorted. When iterative computation is fully implemented until it fully converges, the phase unwrapping tends to fail. In order to achieve the goal of the successful solution, it is necessary to eliminate the noise in the phase data and to
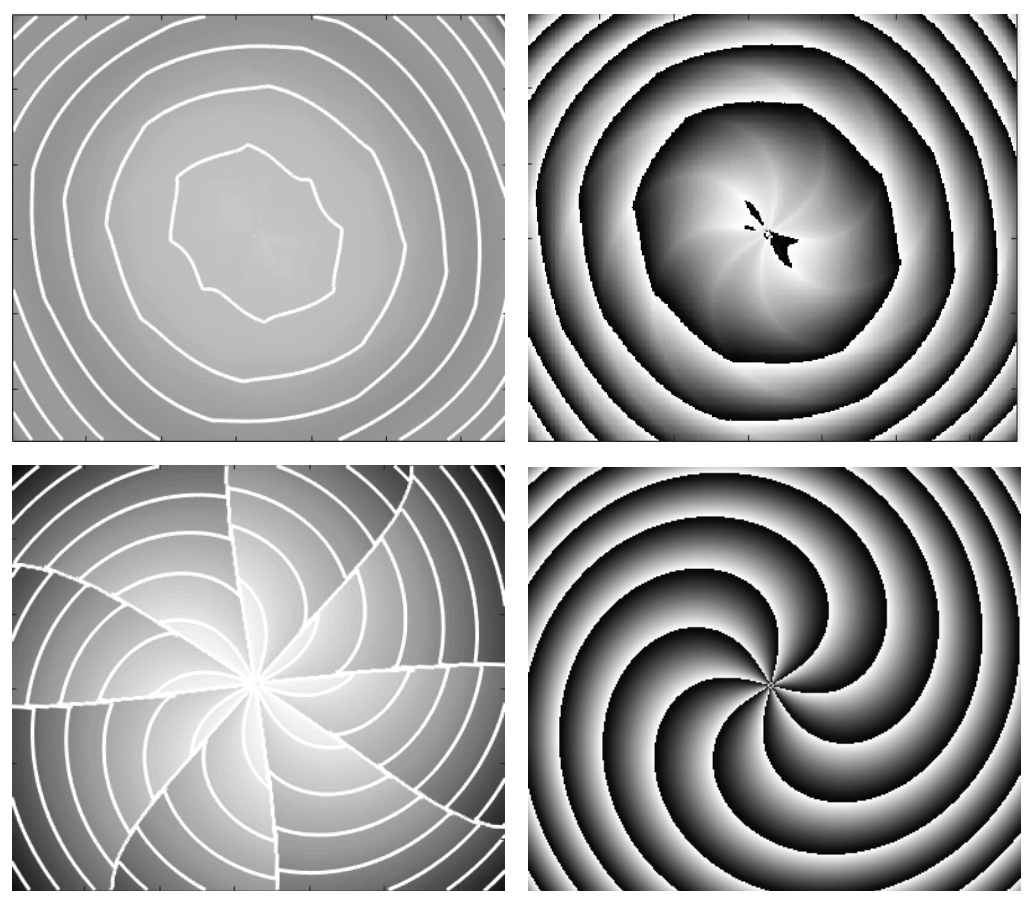

Fig. 3 The comparison of the unwrapping and rewrapping of eight-helix surface. 
modify the surrounding area of the phase image. In other words, it is necessary to remove the noise of large high frequency components. In addition, as shown in the figure, if the pattern of fringes is spirally distributed, the iterative method must be applied to the above algorithm to get the correct results. It is found that the discrete cosine transform based on least square method is the most effective.

\section{Experimental Results and Discussion}

\subsection{Application Interface of SLM Operation}

A system interface for operating SLM is designed with $\mathrm{VC}++$ compiler as shown in Fig. 4. All control mask images were BITMAP format in the gray scale values as change in applied voltage. The file menu includes the mask image open, save, recent used file items. The initial setting function is in the setting items including calibration background reset, and wavelength compensation. First of all, mask images will be changed to in 8bit gray scale. For the BITMAPFILEHEADER, only two items are needed to revise:
bfOffBits $=$ sizeof(BITMAPFILEHEADER $)+\operatorname{sizeof}($ BITMAPFILEHEADER)+256*sizeof(RGBQUAD); bfSize= $=$ fOffBits + nImageSize;

And for the BITMAPINFO, also there are two items needed to revise:

bmiHeader.biBitCount=8;

miHeader.biSizeImage $=$ nImageSize2;

Other is Colormap, also needed to rewrite.

Advantages of SLM operation interface are as following:

- can operate the SLM pixel by pixel from software instead of hard ware operation;

- possible for calibrating the SLM pixel by pixel.

\subsection{SLM Initial Distortion Phase Measurement}

The LCOS reflective SLM under analysis is manufactured by Hamamatsu. The number of pixels is $1,280 \times 1,024$, and the total size is $16 \times 12.8$ square millimeters. The size of each pixel is $12.5 \times 12.5$ square micrometers. The device allows 255 distinct phase levels and a maximum phase variation of more than $2 \pi$ can be generated. So we measured the phase distortion

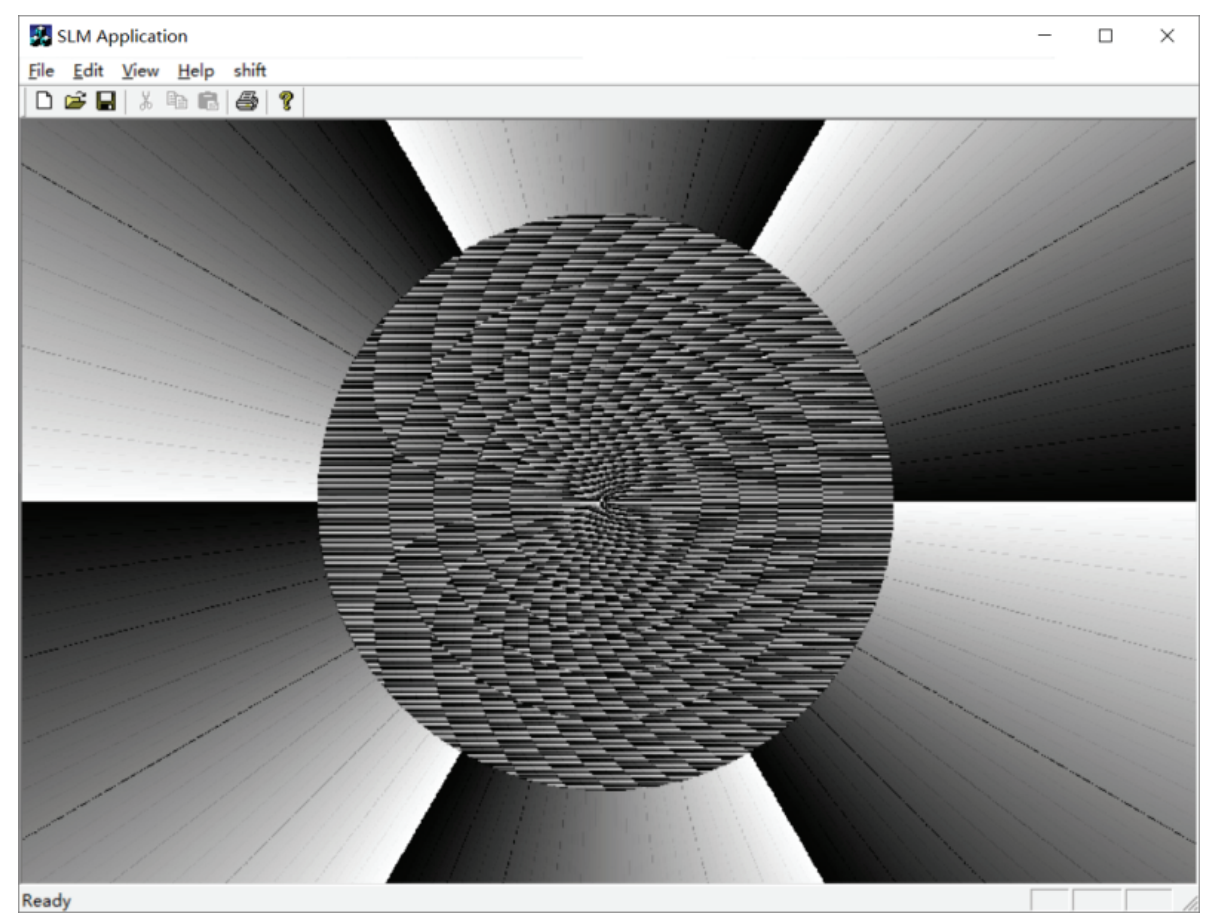

Fig. 4 The application interface of SLM. 
and combined the LUT data provided by manufacture for calibrating the device. Fig. 1 shows the SLM test setup schema. The incident light is aligned at an angle to the optic axis of the LC modulator, which causes the SLM to vary the polarization angle of the reflected light. With this configuration, polarization of the incident light is polarized at a proper angle. One will observe the reflected output light and the interferogram with a large contrast. Adjusting the wave plate, the interference patterns were recorded by CCD camera.

Fig. 5 shows the interference pattern recorded by the 4-step phase-shifting method. The distortion phase can be obtained by using phase equations of four interference patterns (3).

Fig. 6 shows the interference pattern obtained from the carrier interference fringes and the initial distortion phase obtained by the FFT analysis method. When correcting the distortion phase, the photomap of the optical receiving part is measured so as to accurately match the position in pixels (Fig. 7).

For the method of RQPT, we try to use a method, we call it correlation-like analysis, dividing the whole area we want to demodulate, into several sub-windows, performing the optimization for every sub-window using above mentioned algorithm (see Section 3.1), the area in calculating is part overlapping with the undetermined area, using correlation-like analysis, the data to update in undetermined area could be a disambiguation solution. The snapshot of the calculation process is shown in Fig. 8. The experimental results show that the RQPT method has
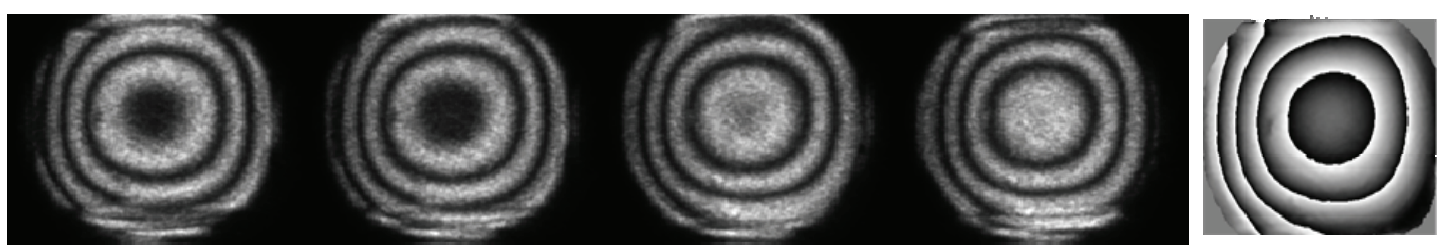

Fig. 5 4-step phase shift interference fringes and wrapped phase of SLM.

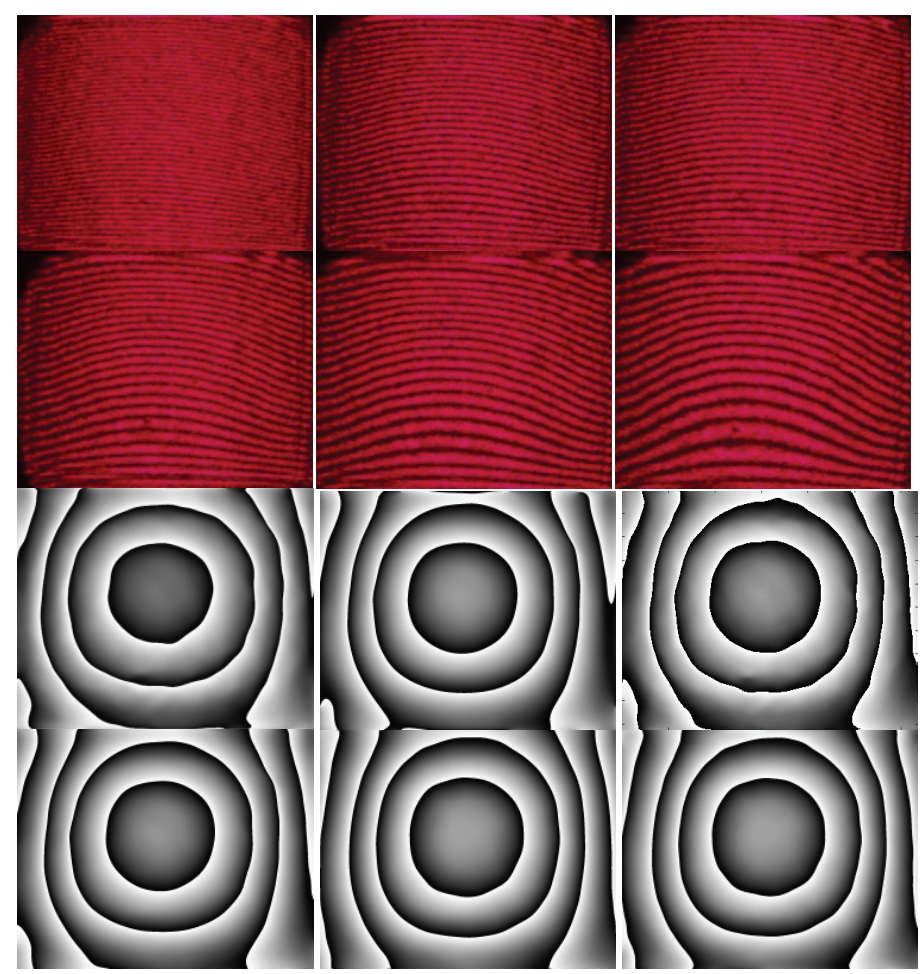

Fig. 6 Interference fringe pattern and phase extraction result. 

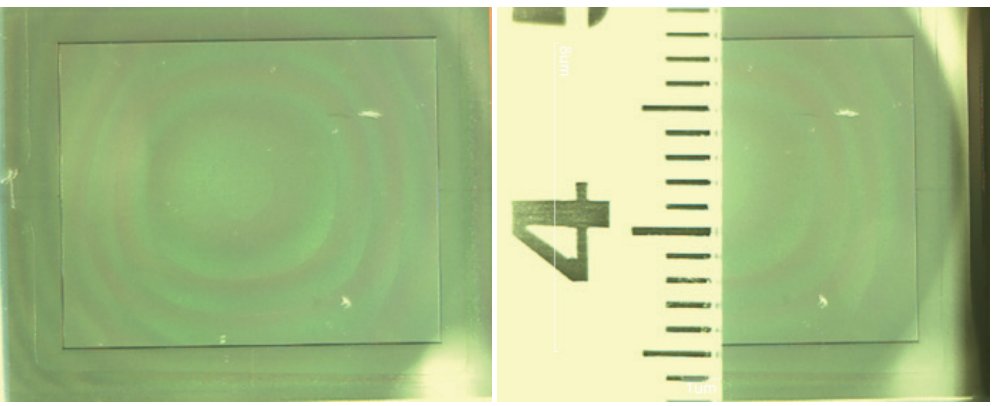

Fig. 7 Photograph of SLM light receiving surface.
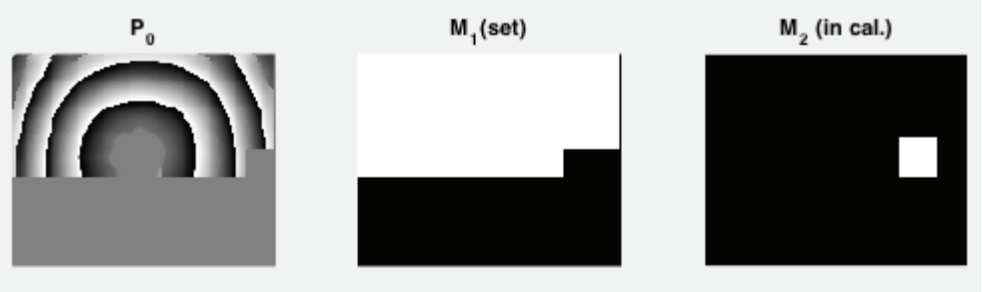

$M_{r}$ (set in cal-area)

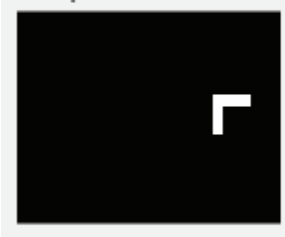

$\mathrm{Mp}_{1}$ (set in cal.)

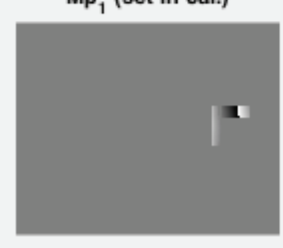

$p_{1}$ (calculated)

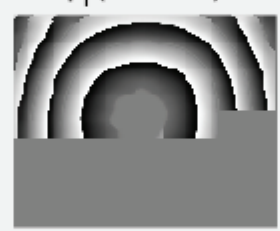

$\mathrm{Mp}_{2}$ (set in cal.)

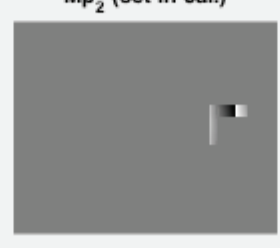

$p_{2}$ (in cal.)

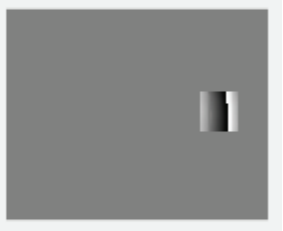

M (in cal.)

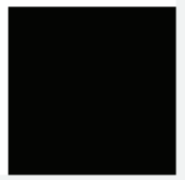

Fig. 8 QPT demodulate closed fringe pattern (sub-window overlap correlation-like method).

the best effect. This can be verified by performance of the correction of the SLM distortion phase, which will be discussed in the next section.

\subsection{SLM initial Distortion Phase Correction}

When using an SLM as a diffraction optical element, because of its initial phase distortion would be included in the $\mathrm{CGH}$ (computer-generated holography) diffraction grating, if the initial phase distortion is loaded into the SLM, interference fringe distortion will be fixed. The calibration result of a light source with a wavelength of 0.658 microns is shown in Fig. 9.

The first row is the interferogram with phase distortion, and the second row is the interferogram after distortion correction. For distorted interference fringes with larger spacing (row 3), the calibration results are shown in Row 4.

\subsection{Correction of SLM Wavelength Dispersion}

In order to observe the change of the interference fringes with wavelength, a phase mask with comparability of gray level is adopted. This mask is shown in Fig. 10, with the phase of the left half (or lower part) unchanged, and the phase of the right half (or upper part) is gradual from 0 to 360 degrees.

For a configuration of which the phase jump line is 

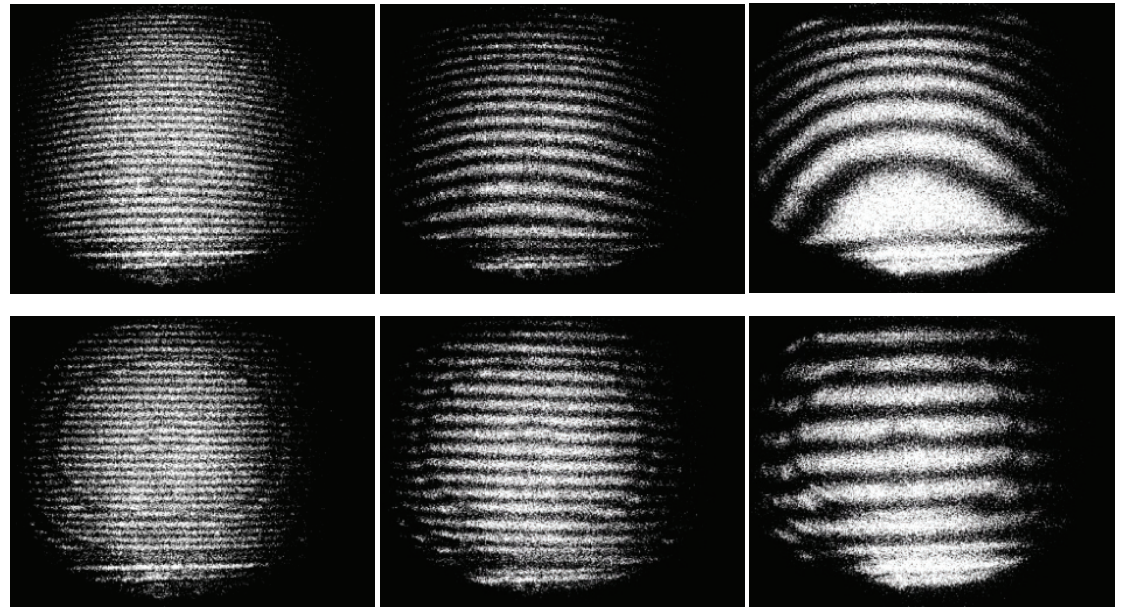

(a) Interference fringes with smaller spacing, calibration effect.
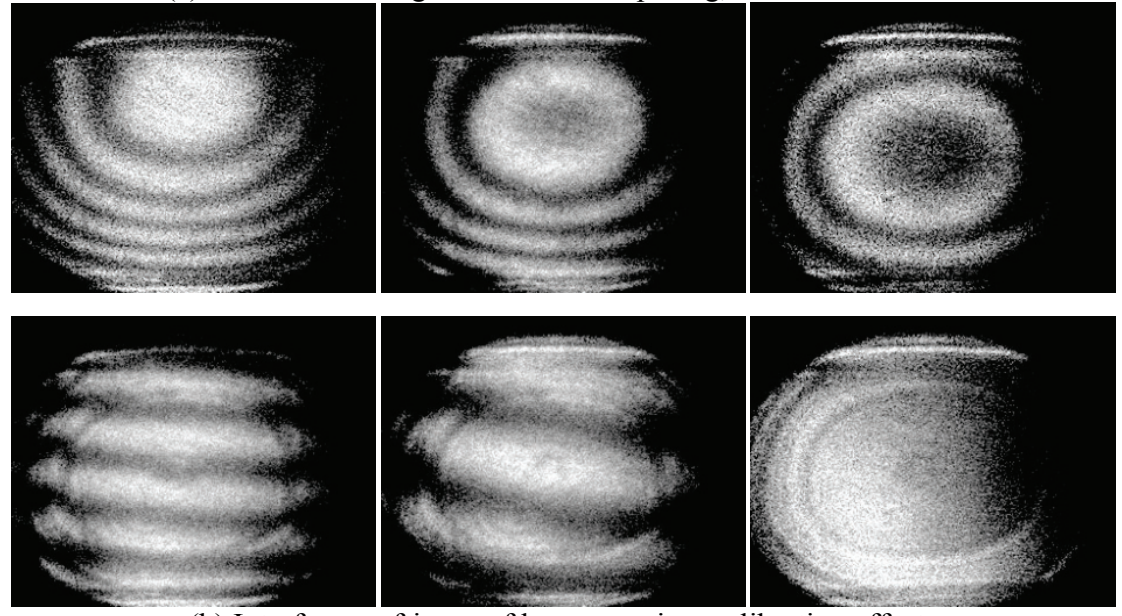

(b) Interference fringes of larger spacing, calibration effect.

Fig. 9 Confirmation of calibration effect (wavelength $0.658 \mu \mathrm{m}$ ).

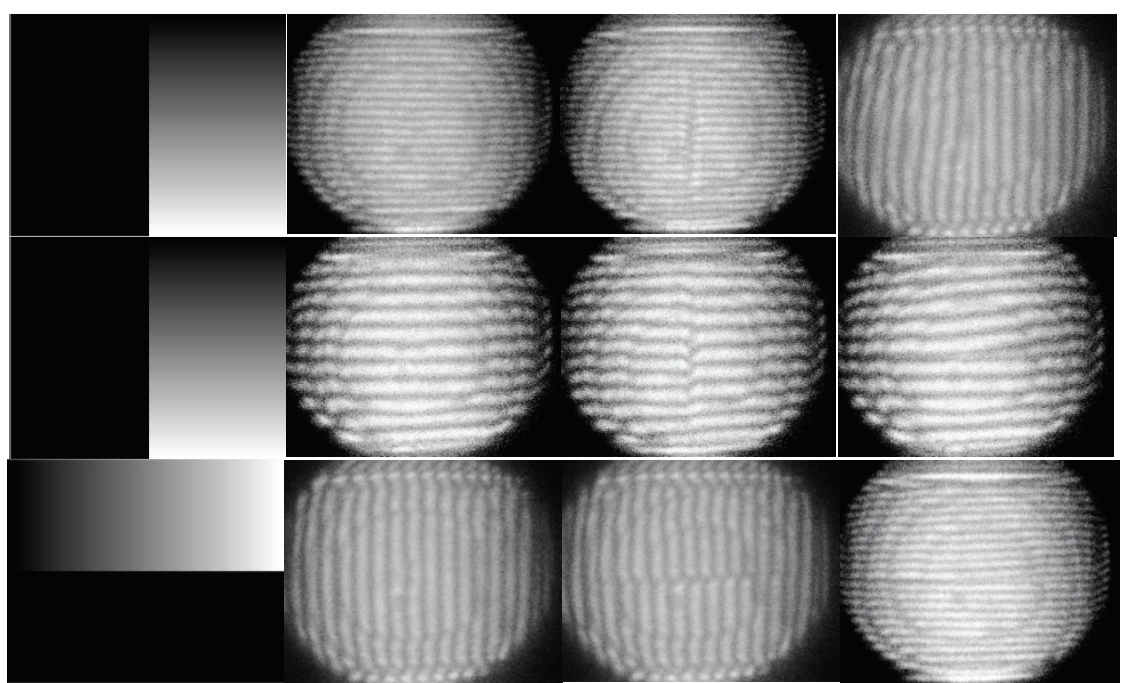

Fig. 10 Experimental result of SLM wavelength dispersion.

perpendicular to the interference fringes, if the maximum phase modulation is 360 degrees, it will be found that the numbers of the interference fringes where the phase is gradual will be one more than one 
where the phase is constant, and the maximum dislocation of interference fringes will locate in the center of the interferogram.

For SLMs with a calibration wavelength of $633 \mathrm{~nm}$, if the wavelength used is greater than this reference wavelength, there will be more than one interference fringe, and if the wavelength used is less than this reference wavelength, there will be no more than one increase in interference fringes in the phase-graded region.

On the other hand, when the phase modulation maximum of the spatial light modulator is PI, the maximum dislocation of interference fringes will appear on the outer edge of the interferogram.

The wavelength dispersion can be compensated well according to the displacement of interference fringes caused by dispersion of different wavelength.

In addition, the wavelength disperse compensation can also be achieved by using the LUT data divided by manufacture. Fig. 11 shows the interfereogram before and after compensation. It is obviously the initial wave front was compensated successfully.

\subsection{Diffraction by Blazed Kinoform: The Generation of} Lageere-Gaussian Beam

Fig. 12 shows the distortion phase and two types of LG mask. Fig. 13 shows the effect of correcting the distortion phase when binary mask and phase modulation mask are used for phase modulation SLM. Fig. 14 shows a photograph of the intensity distribution of the vortex light wave generated by the LG beam kinoform. From left, they are LG beam pattern without, and with distortion initial phase calibration as well as the interference between the order of +1 and -1 . This is the first-order diffracted light from the kinoform mask in the case of mode index $\mathrm{p}$ and 1 both of 5 , that is, mode $\mathrm{LG}_{5}^{5}$.

In Fig. 15, it shows LG beam profile, comparison between theoretical value and experimental value.

In the case where the parameter $\mathrm{R}$ shows the correlation between the measured value and the theoretical value it is defined by the sum of the square of the residual,

$$
\mathrm{Q}=\sum\left(\mathrm{y}-\mathrm{y}^{*}\right)^{2}
$$

and the sum of the squared values, $\sum y^{2}$.

$$
\mathrm{R}=1-\sqrt{\mathrm{Q} / \sum \mathrm{y}^{2}}
$$

The parameter $\mathrm{R}$ representing the correlation, gives $\mathrm{R}=0.6596$. Here, $\mathrm{y}$ and $\mathrm{y}^{*}$ represent measured values and estimated values, respectively. The correlation coefficient between the experimental value and the theoretical value was found to be 0.9417 . As shown in Fig. 15, it can be seen that the experimental results are in good agreement with the theoretical analysis.

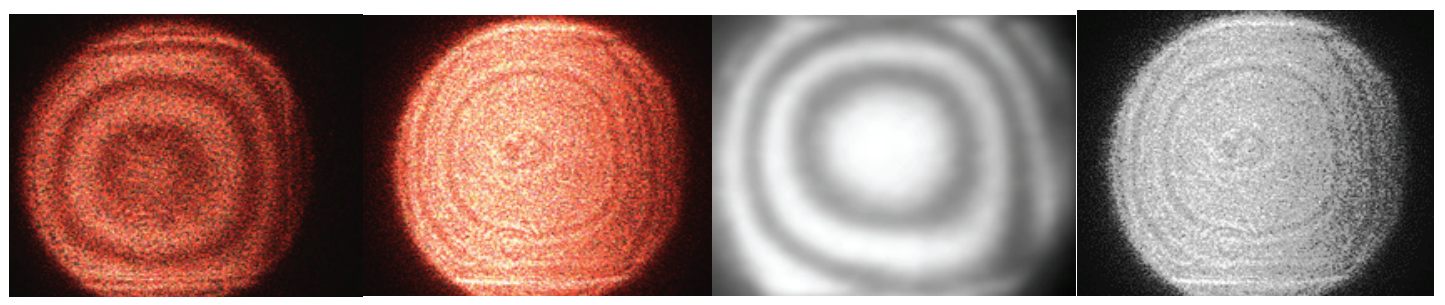

Fig. 11 Phase compensation result.

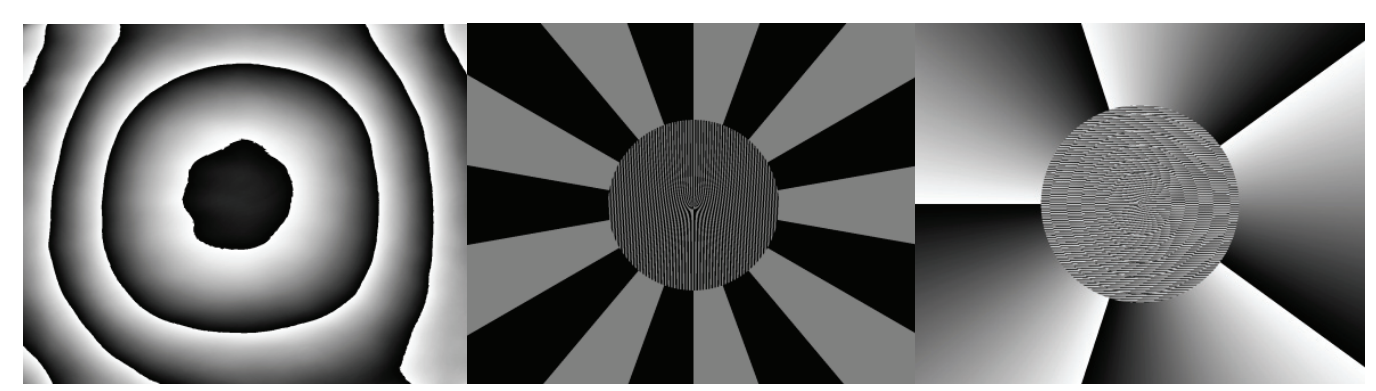

Fig. 12 Distortion phase and LG mask. 
Fig. 13 Distortion corrected diffraction mask.
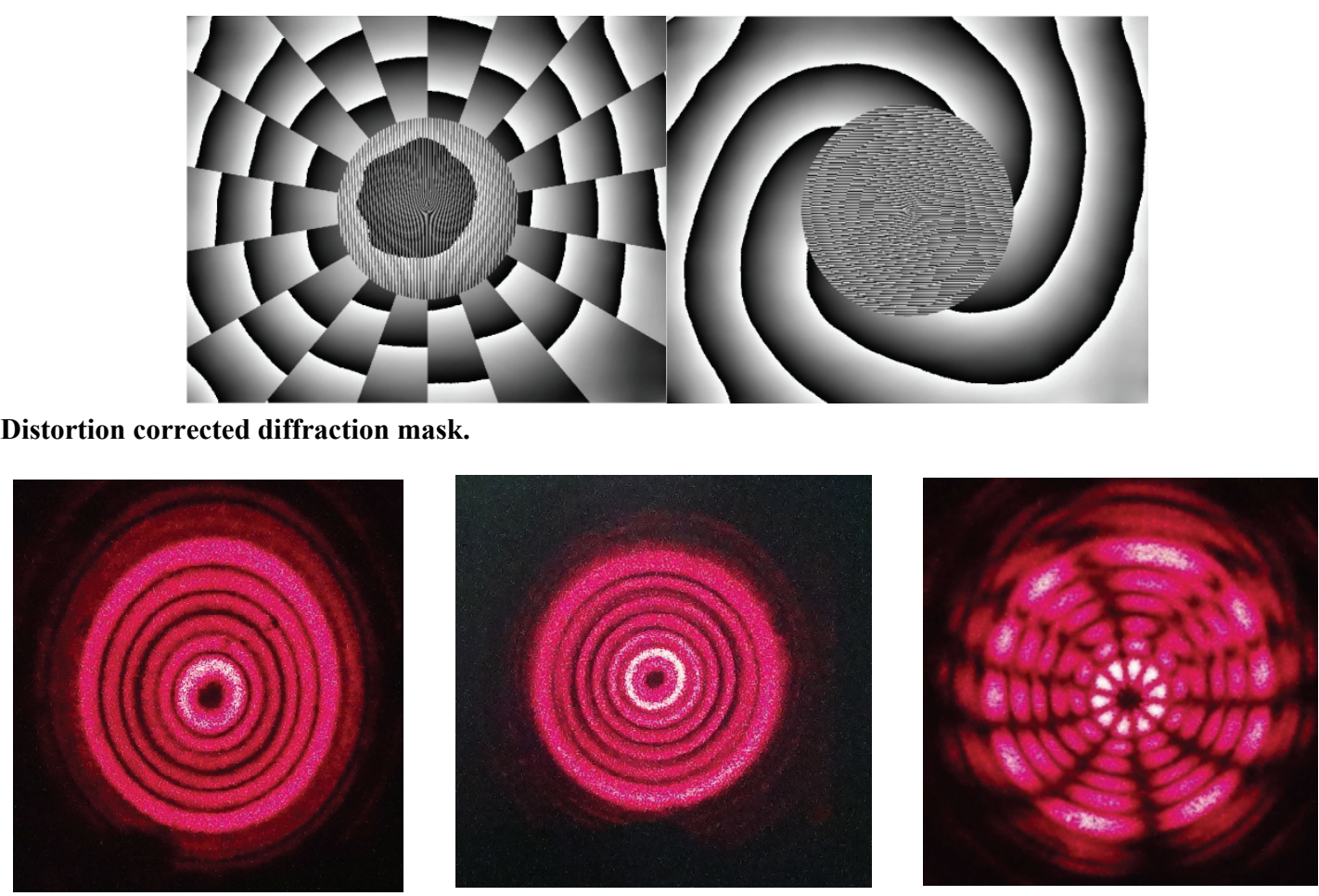

Fig. 14 LG beam pattern, comparison between theoretical value and experimental value.

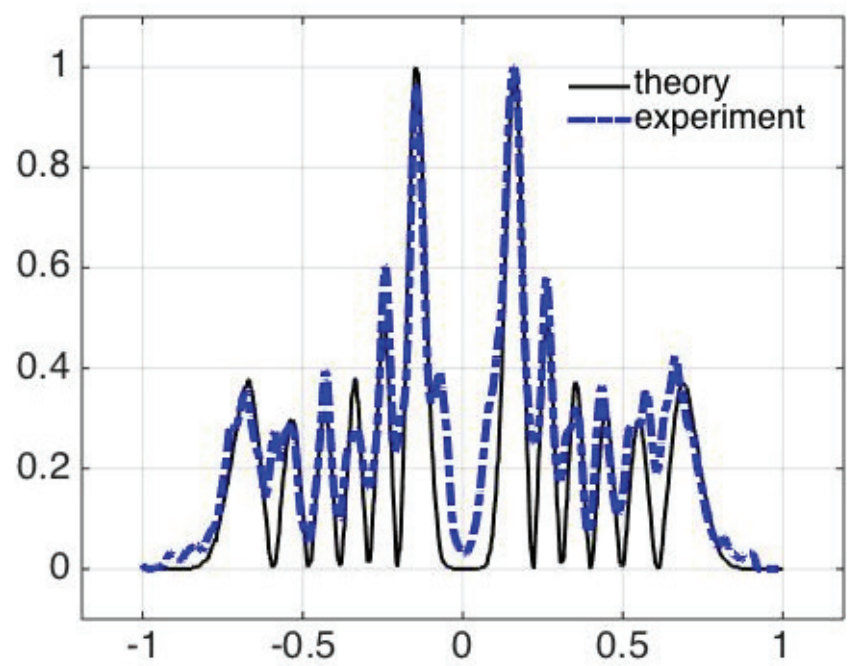

Fig. 15 LG beam profile, comparison between theoretical value and experimental value.

\section{Conclusion}

The initial phase distortion caused by manufacture process and silicon substrate surface error will largely affect the phase modulation effect of device. We have measured this initial phase distortion with reflective interferometer technique. For characterizing properties of modulation and operating the device, the system interface for operating SLM is designed with $\mathrm{VC}++$ compiler. With this system, the initial distortion phase is determined by measuring the reflective interference and modulation of device is compensated by using our system.

For phase extraction from an interferogram, by using the method of phase shifting or the method of Fourier transform, it was found that the phase 
distortion although initial distortion and dispersion correction of SLM are initially solved, there are some deviations. The reasons can be as follows:

(1) For the four-step phase shift method, it is necessary to record more than 4 interferograms, and the output power of the laser light source will change during the measurement process, resulting in a large measurement error. At the same time, the mechanical vibration of the measurement system cannot be ignored.

(2) Even in the case of carrier interference fringes, the wavefront tilt of the reference interference light is usually limited, and the bandwidth of the measured bands is limited to a large extent. That is to say, because the fringe analysis method is on the surface of the object under test, concave and convex change frequency is lower than the frequency of the carrier fringes enough premise established, so the high frequency component will become less sensitive.

For this reason, an improved RQPT method was employed for demodulating the fringes pattern. It was shown that the RQPT method has the best effect. The advantages of SLM operation interface provided in this study are as followings:

(1) It can operate the SLM pixel by pixel from software instead of hardware operation.

(2) It is possible to calibrate the SLM pixel by pixel.

According to the displacement of interference fringes caused by different wavelength dispersion, the wavelength dispersion can be well compensated. Another way to calibrate wavelength dispersion is to use the LUT data provided by the manufacturer to compensate for the dispersion of the wavelength. In practice, it is also found to be a very effective method.

The correction effect of the distortion phase is verified by using the diffraction of computer-generated holographic diffraction to produce LG beam. It can be seen that the SLM diffraction after correction of phase distortion, the resulting $\mathrm{LG}$ beam is in good agreement with the theoretical expectation.

\section{References}

[1] Kanghua, L., and Bahaa, E. A. Saleh, 1991. "Complex Amplitude Reflectance of the Liquid Crystal Light Valve." Applied Optics 30 (17): 2354.

[2] Servin, M., Marroquin, J. L., and Cuevas, F. J. 1997. "Demodulation of a Single Interferogram by Use of a Two-Dimensional Regularized Phase-Tracking Technique." Appl. Opt. 36: 4540-8.

[3] Tian, C., Yang, Y., Liu, D., Luo, Y., and Zhuo, Y. 2010. "Demodulation of a Single Complex Fringe Interferogram with a Path-Independent Regularized Phase-Tracking Technique.” Appl Opt. 49 (2): 170-9.

[4] Kai, L., and Kemao, Q. 2012. "A Generalized Regularized Phase Tracker for Demodulation of a Single Fringe Pattern." Opt. Express 20 (11): 12579-92.

[5] Schofield, M. A., and Zhu, Y. 2003. "Fast Phase Unwrapping Algorithm for Interferometric Applications." Opt. Lett. 28 (14): 1194-6.

[6] Shi, W., Zhu, Y., and Yao, Y. 2010. "Discussion about the DCT/FFT Phase-Unwrapping Algorithm for Interferometric Applications." Optik 121: 1443-9.

[7] Volkov, V. V., and Zhu, Y. 2003. "Deterministic Phase Unwrapping in the Presence of Noise." Opt. Lett. 28 (22): 2156.

[8] Jeught, S., Sijbers, J., and Dirckx, J. 2015. "Fast Fourier-Based Phase Unwrapping on the Graphics Processing Unit in Real-Time Imaging Applications.” $J$. Imaging 1: 31-44.

[9] Hui, M., Wang, D. S., Li, Q. X., Deng, N. M., and Xu, Y. X. 2003. "Phase Unwrapping Method Based on the Solution of Discrete Poisson Equation." ACTA Optica Sinica 23 (10): 1246. 